\title{
Improved Strong Tracking Cubature Kalman Filter for Target Tracking
}

\author{
Shuida Bao ${ }^{1}$ anf An Zhang, ${ }^{2, *}$ \\ ${ }^{1}$ School of Electronics and Information, Northwestern Polytechnical University, Xi'an 710072, China \\ ${ }^{2}$ School of Aeronautics, Northwestern Polytechnical University, Xi'an 710072, China
}

\begin{abstract}
Cubature Kalman filter (CKF) is a very popular nonlinear filter algorithm recently. CKF obtains better numerical stability and accuracy in high dimensional situation compared to UKF. However, in case of process model uncertainty, the performance of CKF will greatly degrade or even provoke divergence. An improved strong tracking CKF (ISTCKF) is proposed to keep the numerical stability and improve the robustness. First, the theoretical framework of strong tracking filter (STF) is combined with CKF. Then, an enhanced fault detection and isolation technique is established to overcome the drawback in STCKF. ISTCKF only performs correction phase when the process model uncertainty is detected and isolated. The ISTCKF is tested and validated via a target tracking model.
\end{abstract}

Keywords-cubature Kalman filter; strong tracking filter; fault detection and isolation; numerical stability

\section{INTRODUCTION}

Kalman filter (KF) has been widely used as a linear system filtering algorithm since it was proposed. KF has two important advantage: (1) it utilizes the recursive form; (2) it is designed in time domain. However, the world is non-linear in nature, even for some linear system is the approximation of non-linear system simply. KF will fail if the system function is non-linear.

Many non-linear filter algorithms have been proposed by researchers, such as extended Kalman filter[1] (EKF), Unscented Kalman filter[2] (UKF) and cubature Kalman filter[3; 4] (CKF), etc. At present, almost all non-linear filter algorithms utilize the recursive form and are designed in time domain, following the design ideas of KF. EKF applies the first order Taylor series expansion of non-linear function to approximate the non-linear system as a linear system, and then handle the system with the framework of KF. UKF is proposed in light of the intuition that it is much easier to approximate a probability distribution than an arbitrary non-linear transformation. Unscented transform is the core of UKF, which use a set of chosen sample points to represent the probability distribution. Compared with EKF, UKF provides more accurate performance and omits the cumbersome operations like derivation and Jacobi matrices. However, UKF encounters the numerical stability problem as utilized in high-dimensional system, because the stem at the center (the mean) of the sigma points owns more weight which is usually negative in highdimensional system. CKF is proposed with the spherical-radial cubature rule. Different from UKF, CKF is obtained through strict mathematical derivation. In fact, CKF could be regard as a special UKF, when the coefficient for the sigma point in the center is set as $\kappa=0$. Obviously CKF overcomes the drawback of UKF, and gets better performance in highdimensional system.

However, CKF still does not obtain the adaptability to the uncertainty in process model. The process model uncertainty will affect the filtering performance or even lead to divergence[5]. Covariance matching techniques have been proved to CKF and UKF to build adaptive CKF[6] and adaptive UKF, which possess better adaptability and robustness Strong tracking filter[7] is an innovation covariance matching based adaptive EKF with a time-varying suboptimal fading factor. STF effectively solves the drawback of EKF when the process model is uncertain.

In this paper, the theoretical framework of STF is applied to CKF. The shortcoming of fault detection method used in STF is analyzed. Then an enhanced fault detection and isolation method is built, and the improved strong tracking CKF (ISTCKF) is established. The proposed algorithm is tested with a target tracking model.

\section{IMPROVED STRONG TRACKING CUBATURE KALMAN} FILTER

\section{A. CKF Algorithm}

Considering the following non-linear discrete-time system with additive noises:

$$
\begin{gathered}
\boldsymbol{x}_{k}=f\left(\boldsymbol{x}_{k-1}\right)+\boldsymbol{w}_{k-1} . \\
\boldsymbol{z}_{k}=h\left(\boldsymbol{x}_{k}\right)+\boldsymbol{v}_{k} .
\end{gathered}
$$

where $\boldsymbol{x}_{k} \in \mathbf{R}^{n}$ is the system state vector, $\boldsymbol{z}_{k} \in \mathbf{R}^{m}$ is the measurement vector; $f(\square)$ and $h(\square)$ are the process function and the measurement function respectively; $\boldsymbol{w}_{k-1}$ and $\boldsymbol{v}_{k}$ are white noise with zero mean and covariance $\boldsymbol{Q}$ and $\boldsymbol{R}$, respectively.

\section{- Time Update}

Assume at instant $k$, the posterior density function $p\left(\boldsymbol{x}_{k-1} \mid \boldsymbol{D}_{k-1}\right)=N\left(\hat{\boldsymbol{x}}_{k-1 / k-1}, \boldsymbol{P}_{k-1 / k-1}\right)$ is known. The time update equations are given by 


$$
\begin{gathered}
\boldsymbol{P}_{k-1 / k-1}=\boldsymbol{S}_{k-1 / k-1} \boldsymbol{S}_{k-1 / k-1}^{T} . \\
\boldsymbol{X}_{i, k-1 / k-1}=\boldsymbol{S}_{k-1 / k-1} \boldsymbol{\xi}_{i}+\hat{\boldsymbol{x}}_{k-1 / k-1} . \\
\boldsymbol{X}_{i, k / k-1}^{*}=f\left(\boldsymbol{X}_{i, k-1 / k-1}\right) . \\
\hat{\boldsymbol{x}}_{k / k-1}=\frac{1}{l} \sum_{i=1}^{l} \boldsymbol{X}_{i, k / k-1}^{*} . \\
\boldsymbol{P}_{k / k-1}=\frac{1}{l} \sum_{i=1}^{l} \boldsymbol{X}_{i, k / k-1}^{*} \boldsymbol{X}_{i, k / k-1}^{* T}-\hat{\boldsymbol{x}}_{k / k-1} \hat{\boldsymbol{x}}_{k / k-1}^{T}+\boldsymbol{Q}_{k-1} .
\end{gathered}
$$

\section{- Measurement Update}

The measurement update equations are given by

$$
\begin{gathered}
\boldsymbol{P}_{k / k-1}=\boldsymbol{S}_{k / k-1} \boldsymbol{S}_{k / k-1}^{T} . \\
\boldsymbol{X}_{i, k / k-1}=\boldsymbol{S}_{k / k-1} \boldsymbol{\xi}_{i}+\hat{\boldsymbol{x}}_{k / k-1} . \\
\boldsymbol{Z}_{i, k / k-1}=h\left(\boldsymbol{X}_{i, k / k-1}\right) . \\
\boldsymbol{\boldsymbol { z }}_{k z / k-1}=\frac{1}{l} \sum_{i=1}^{l} \boldsymbol{Z}_{i, k / k-1}^{*} . \\
\frac{1}{l} \sum_{i=1}^{l} \boldsymbol{Z}_{i, k / k-1} \boldsymbol{Z}_{i, k / k-1}^{T}-\hat{\boldsymbol{z}}_{k / k-1} \hat{\boldsymbol{z}}_{k / k-1}^{T}+\boldsymbol{R}_{k} . \\
\boldsymbol{P}_{k / k}=\boldsymbol{P}_{k / k-1}-\boldsymbol{K}_{k} \boldsymbol{P}_{z z, k / k-1} \boldsymbol{K}_{k}^{T} . \\
\hat{\boldsymbol{x}}_{k / k}=\hat{\boldsymbol{x}}_{k / k-1}+\boldsymbol{K}_{k}\left(\boldsymbol{z}_{k}-\hat{\boldsymbol{z}}_{k / k-1}\right) . \\
\sum_{i=1}^{l} \boldsymbol{X}_{i, k / k-1} \boldsymbol{Z}_{i, k / k-1}^{T}-\hat{\boldsymbol{x}}_{k / k-1} \hat{\boldsymbol{z}}_{k / k-1}^{T} . \\
\boldsymbol{K}_{k}=\boldsymbol{P}_{x z, k / k-1} \boldsymbol{P}_{z z, k / k-1}^{-1} .
\end{gathered}
$$

\section{B. Strong Tracking Cubature Kalman Filter}

The basic concept of STF is orthogonal principle, which can be mathematically depicted as follow

$$
\boldsymbol{V}_{j, k}=E\left[\boldsymbol{e}_{k} \boldsymbol{e}_{k+j}\right]=0
$$

where $\boldsymbol{V}_{j, k}$ is the auto-covariance, $j \neq 0$; $\boldsymbol{e}_{k}$ is the innovation sequence, $\boldsymbol{e}_{k}=\boldsymbol{z}_{k}-\hat{\boldsymbol{z}}_{k / k-1}$.

If the process model is accurate, (17) is satisfied. If not, the suboptimal fading factor is applied to the predicted error covariance $\boldsymbol{P}_{k / k-1}$, in order to satisfy the (17). When it comes to $\mathrm{CKF}$, the suboptimal fading factor $\lambda_{k}$ can be introduced as follow,

$$
\boldsymbol{P}_{k / k-1}=\lambda_{k}\left(\frac{1}{l} \sum_{i=1}^{l} \boldsymbol{X}_{i, k / k-1}^{*} \boldsymbol{X}_{i, k / k-1}^{* T}-\hat{\boldsymbol{x}}_{k / k-1} \hat{\boldsymbol{x}}_{k / k-1}^{T}\right)+\boldsymbol{Q}_{k-1} .
$$

$\lambda_{k}$ is calculated as follows:

$$
\lambda_{k}=\max \left(1, \mathrm{C}_{k}\right)
$$

$$
C_{k}=\frac{\operatorname{tr}\left(\boldsymbol{N}_{k}\right)}{\operatorname{tr}\left(\boldsymbol{M}_{k}\right)}
$$

$$
\boldsymbol{N}_{k}=\boldsymbol{V}_{k}-\boldsymbol{R}_{k}-\left[\boldsymbol{P}_{z z, k / k-1}^{o}\right]^{T}\left[\left(\boldsymbol{P}_{k / k-1}^{o}\right)^{-1}\right]^{T} \boldsymbol{Q}_{k-1}\left[\boldsymbol{P}_{k / k-1}^{o}\right]^{-1} \boldsymbol{P}_{z z, k / k-1}^{o}
$$

$$
\boldsymbol{M}_{k}=\boldsymbol{P}_{z z, k / k-1}^{o}-\boldsymbol{V}_{k}+\boldsymbol{N}_{k}
$$

where $\operatorname{tr}(\square)$ is the trace of the related matrix, the superscript 'o' denotes the variable before $\lambda_{k}$ is introduced. $\boldsymbol{V}_{k}$ is the practical innovation covariance, which is evaluated as

$$
\boldsymbol{V}_{k}=\left\{\begin{array}{l}
\boldsymbol{e}_{1} \boldsymbol{e}_{1}^{\mathrm{T}}, k=0 \\
\frac{\rho \boldsymbol{V}_{k-1}+\boldsymbol{e}_{k} \boldsymbol{e}_{k}^{\mathrm{T}}}{1+\rho}, k>0
\end{array} .\right.
$$

where $\rho$ is the forgetting factor.

\section{Enhanced Fault Detection and Isolation}

In strong tracking theory, $\lambda_{k}$ is derived by keeping the theoretical innovation covariance consistent with the practical innovation covariance. 


$$
\boldsymbol{P}_{z z, k / k-1}=\boldsymbol{V}_{k} .
$$

According to Kalman filtering theory, innovation covariance $\boldsymbol{e}_{k}$ is a Gaussian distribution with mean 0 and variance $\boldsymbol{P}_{z z, k / k-1}$. $\boldsymbol{e}_{k}$ at time $k$ can be regard as a sampling of theoretical innovation covariance. As show in (23), $\boldsymbol{V}_{k}$ is calculated by fading memory technique with $\boldsymbol{e}_{k} \boldsymbol{e}_{k}^{\mathrm{T}}$. For convenience, $\boldsymbol{V}_{k}$ is regard as the practical innovation covariance in this paper.

In STF, $\lambda_{k}$ is also utilized as the fault detection criterion. $\lambda_{k}>1$ means there is some kind of uncertainties in process model. $\lambda_{k} \leq 1$ means the process model is accurate. In the light of probability theory, the probability of $\lambda_{k}>1$ is

$$
P\left(\boldsymbol{V}_{k}>\boldsymbol{P}_{z z, k / k-1}\right)=0.3474
$$

Equation (25) indicates that even if when the process model is accurate, STF has a quite large probability treating as a fault.

Based on above analysis, $\lambda_{k}$ is not an excellent criterion for fault detection in STCKF. Another widely used criterion is derived to use as fault detection in ISTCKF. Consequently, $\lambda_{k}$ only calculates when relative fault is detected, and in all other cases, ISTCKF works as the CKF.

Two hypotheses are defined:

$\gamma_{0}$ : The system is normally operating.

$\gamma_{1}$ : There is some kind process model uncertainty.

As aforementioned, $\boldsymbol{e}_{k}$ is a Gaussian distribution, then $\beta_{k}$ is a $\chi^{2}$ distribution with $m$ degree of freedom.

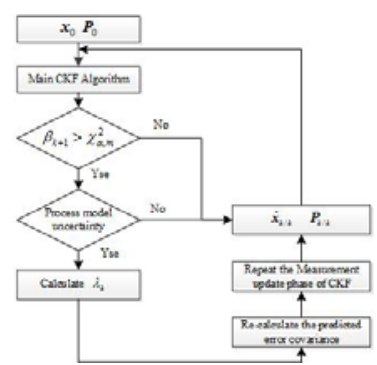

FIGURE I. ARCHITECTURE OF THE PROPOSED ISTCKF

$$
\beta_{k}=\boldsymbol{e}_{k}^{T}\left[\boldsymbol{P}_{z z, k / k-1}\right]^{-1} \boldsymbol{e}_{k} .
$$

A certain level of significance, $\alpha$, is chosen,

$$
P\left\{\chi^{2}>\chi_{\alpha, m}^{2}\right\}=\alpha, 0<\alpha<1 .
$$

Then a threshold value $\chi_{a, m}^{2}$ is obtained. $\beta_{k}$ will be greater than $\chi_{a, m}^{2}$, if hypothesis $\gamma_{1}$ is established, namely

$$
\begin{aligned}
& \gamma_{0}: \beta_{k} \leq \chi_{\alpha, m}^{2} \quad \forall k \\
& \gamma_{1}: \beta_{k}>\chi_{\alpha, m}^{2} \quad \exists k
\end{aligned}
$$

It is notable that suboptimal fading factor can only deal with uncertainty in the process model. Another key problem is to identify the fault type (a measurement malfunction or process model uncertainty). The fault isolation can be realized by an algorithm similar to the one proposed for aircrafts[8]. Based on the Doyle-Stein condition, A similar robust CKF is built to isolate the process model uncertainty and measurement malfunction. If the process noise covariance matrix is set as

$$
\boldsymbol{Q}_{(q)}=\boldsymbol{Q}+q^{2} \boldsymbol{B} \boldsymbol{V} \boldsymbol{B}^{T}
$$

Where $q$ approaches to infinity, $\boldsymbol{V}$ is any positive definite symmetric matrix, $\boldsymbol{B}$ is the control distribution matrix in continuous time, then the robust CKF is acquired. The overall scheme for the ISTCKF is shown in Figure 1.

\section{SimUlation}

The ISTCKF is tested via a target tracking model[6]. The non-linear process equation of the target tracking is modeled as below:

$$
\boldsymbol{x}_{k}=\left[\begin{array}{ccccc}
1 & \frac{\sin \Omega(k) T}{\Omega(k)} & 0 & \frac{-1+\cos \Omega(k) T}{\Omega(k)} & 0 \\
0 & \cos \Omega(k) T & 0 & -\sin \Omega(k) T & 0 \\
0 & \frac{1-\cos \Omega(k) T}{\Omega(k)} & 1 & \frac{\sin \Omega(k) T}{\Omega(k)} & 0 \\
0 & \sin \Omega(k) T & 0 & \cos \Omega(k) T & 0 \\
0 & 0 & 0 & 0 & 1
\end{array}\right] \boldsymbol{x}_{k-1}+\boldsymbol{w}_{k-1} .
$$

where $\boldsymbol{x}_{k}$ is the state of the aircraft and $\boldsymbol{x}_{k}=\left[x_{k} \dot{x}_{k} y_{k} \dot{y}_{k} \Omega_{k}\right]$. $x_{k}$ and $y_{k}$ are the position of aircraft in the coordinate axis. Their derivative $\dot{x}_{k}$ and $\dot{y}_{k}$ are the velocity in the corresponding axis. $\Omega_{k}$ is the turning rate. $T$ is the timeinterval of simulation and $T=1 . \boldsymbol{w}_{k-1}$ is white noise with zero mean and covariance $\boldsymbol{Q}$.

The radar is placed at $(0,0)$, which can apply the range $\sigma$ and the bearing $\theta$.

$$
\left[\begin{array}{c}
\sigma_{k} \\
\theta_{k}
\end{array}\right]=\left[\begin{array}{c}
\sqrt{x_{k}^{2}+y_{k}^{2}} \\
\operatorname{atan} 2\left(y_{k}, x_{k}\right)
\end{array}\right]+\boldsymbol{v}_{k} .
$$

Where atan2 is the four-quadrant inverse tangent function, $v_{k}$ is white noise with zero mean and covariance $\boldsymbol{R}$. 
$\boldsymbol{Q}$ and $\boldsymbol{R}$ are set as

$$
\boldsymbol{Q}=\left[\begin{array}{ccccc}
\frac{\varphi_{1} T^{3}}{3} & \frac{\varphi_{1} T^{2}}{2} & 0 & 0 & 0 \\
\frac{\varphi_{1} T^{2}}{2} & \varphi_{1} T & 0 & 0 & 0 \\
0 & 0 & \frac{\varphi_{1} T^{3}}{3} & \frac{\varphi_{1} T^{2}}{2} & 0 \\
0 & 0 & \frac{\varphi_{1} T^{2}}{2} & \varphi_{1} T & 0 \\
0 & 0 & 0 & 0 & \varphi_{2} T
\end{array}\right]
$$

where $\varphi_{1}$ and $\varphi_{2}$ are the scalar variables and $\varphi_{1}=1 \mathrm{~m}^{2} \mathrm{~s}^{-3}$, $\varphi_{2}=1.75 \times 10^{-3} \operatorname{rad}^{2} \mathrm{~s}^{-3}$.

$$
\boldsymbol{R}=\operatorname{diag}\left[1000 \mathrm{~m}^{2} \quad 100 \mathrm{mrad}^{2}\right]
$$

The reference data is generated using

$$
\boldsymbol{x}_{0}=\left[1000 \mathrm{~m} 300 \mathrm{~ms}^{-1} 1000 \mathrm{~m} 0 \mathrm{~ms}^{-1}-3^{\circ} \mathrm{s}^{-1}\right]^{\mathrm{T}} \text {. }
$$

$\boldsymbol{P}_{0}=\operatorname{diag}\left[100 \mathrm{~m}^{2} 10 \mathrm{~m}^{2} \mathrm{~s}^{-2} 100 \mathrm{~m}^{2} 10 \mathrm{~m}^{2} \mathrm{~s}^{-2} 100 \mathrm{mrad}^{2} \mathrm{~s}^{-2}\right]^{\mathrm{T}}$

In the fault detection, $\chi_{\alpha, m}^{2}$ is set as 5.99, which is the threshold value for reliability level $95 \%$ with degree of freedom 2 .

The root mean square error (RMSE) is utilized as a norm to compare the performance with respect to robustness and numerical stability,

$$
\operatorname{RMSE}(i)=\sqrt{\frac{1}{N} \sum_{j=1}^{N}\left(x_{k}-\hat{x}_{k \mid k}\right)^{2}}, i=1 \cdots M .
$$

Where $N$ is the total number of instant, $N=100$, and $M$ is the total Monte Carlo runs, $M=50$.

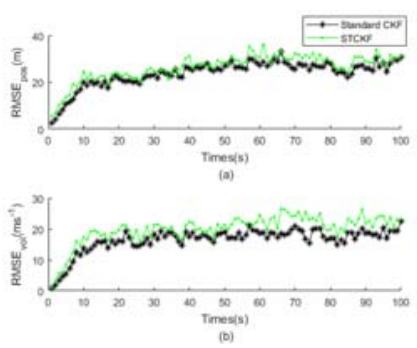

FIGURE II. THE RMSE OF POSITION, VELOCITY FOR STANDARD CKF AND STCKF AT EACH INSTANT

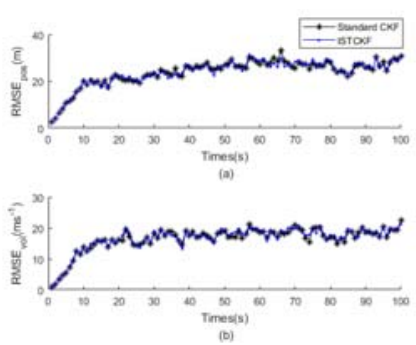

FIGURE III. THE RMSE OF POSITION, VELOCITY FOR STANDARD CKF AND ISTCKF AT EACH INSTANT

\section{A. Normal Situation}

It can be seen from Figure 2 and Figure 3, ISTCKF has better numerical stability than STCKF in normal situation. STCKF owns a large possibility to get wrong judgment for it uses the suboptimal fading factor as the threshold. Due to the $V_{k}$ is not accurate enough for true innovation covariance and the chosen of threshold, STCKF cannot give the best estimation and encounters numerical stability problem when the system is normal. The numerical stability of STCKF is particularly worse in estimating velocity.

In the Monte Carlo simulation, the total number of fault detected by STCKF is 2895. On the other hand, the total number of fault detected by ISTCKF is only 315. The average number of fault detected by ISTCKF in one Monte Carlo is decreased almost 90\%. By using utilizing enhanced fault detection and isolation technique, the numerical stability of ISTCKF is almost equal to CKF.

\section{B. Abrupt State Change}

In this case, an abrupt state change is happened at $k=20$ in every Monte Carlo run.

$$
\left\{\begin{array}{l}
x_{k}=x_{k}+0.08 \times x_{k} \\
y_{k}=y_{k}+0.08 \times y_{k}
\end{array} .\right.
$$

Obviously, ISTCKF and STCKF catch the abrupt state change correctly as shown in Figure 4 and Figure 5. They all go back to normal error range quickly in the position estimation. Meanwhile, CKF takes more than 30 intervals or even longer time in order to go back to normal error range. Paying attention to the velocity estimation, the result of CKF is quite bad after abrupt state change happens. The error even become larger after the position estimation turns to normal. CKF does not have the ability to deal with this fault. In the Monte Carlo simulation, the total number of fault detected by STCKF is 3353, while the total number of fault detected by ISTCKF is only 417. Although the number of fault detected is much lower, the performance of ISTCKF is almost equal with STCKF.

Results show that the suboptimal fading factor enhances robustness of STCKF and ISTCKF. The enhanced fault detection and isolation technique still keeps excellent sensibility with process model uncertainty, although the threshold value is larger than that of STCKF. 


\section{CONCLUSION}

In this paper, an improved strong tracking CKF is proposed. First, STCKF is built with the STF theory. Then, the analysis about STF's fault detection declares that the threshold value is not suitable enough, and causes the numerical stability problem. Therefore, the hypothesis testing theory is applied as fault detection, and a robust CKF is built with Doyle-Stein condition to act as the fault isolation. As a result, ISTCKF only calculates the suboptimal fading factor when the special fault type is detected. When there is no fault detected or other type fault is detected, ISTCKF runs as the normal CKF, and keeps the superior characteristics of CKF. Numerical simulations result show that ISTCKF improves the numerical stability and robustness of CKF.

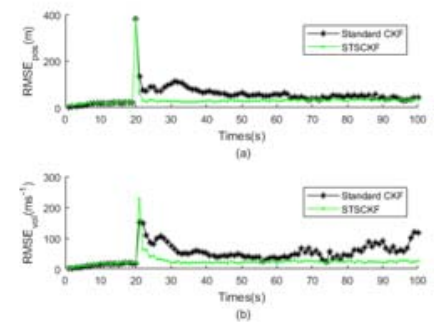

FIGURE IV. THE RMSE OF POSITION, VELOCITY FOR STANDARD CKF AND STCKF IN ABRUPT STATE CHANGE

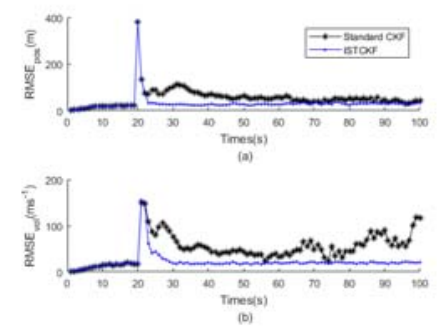

FIGURE V. THE RMSE OF POSITION, VELOCITY FOR STANDARD CKF AND ISTCKF IN ABRUPT STATE CHANGE

\section{ACKNOWLEDGMENT}

This work was supported by the National Science Foundation of China (61573283).

\section{REFERENCES}

[1] G. Y. Kulikov, M. V. Kulikova. "Accurate continuous-discrete extended Kalman filtering for stiff continuous-time stochastic models in chemical engineering, ” Proceedings of European Control Conference, pp. 17281733, 2016.

[2] T. Kodama, A. Okabe, K. Kogiso. "Simultaneous Estimation of Contraction Ratio and Parameter of McKibben Pneumatic Artificial Muscle Model Using Log-Normalized Unscented Kalman Filter," Proceedings of IEEE 4th International Conference on Cyber-Physical Systems, Networks, and Applications, pp. 44-48, 2016.

[3] L. Jiang, B. G. Cai, J. Wang. "Cooperative Localization of Connected Vehicles: Integrating GNSS With DSRC Using a Robust Cubature Kalman Filter," IEEE Transactions on Intelligent Transportation Systems, vol. 99, pp. 1-15, 2016.

[4] I. Arasaratnam, S. Haykin. “Cubature kalman filters,” IEEE Transactions on Automatic Control, vol. 54, pp. 1254-1269, 2009.
[5] J. Zarei, E. Shokri. "Robust sensor fault detection based on nonlinear unknown input observer,” Measurement, vol. 48, pp. 355-367, 2014.

[6] L. Q. ZHAO, J. L. Wang, T. Yu, H. Jian, T. J. Liu. "Design of adaptive robust square-root cubature Kalman filter with noise statistic estimator,"Applied Mathematics and Computation, vol. 256, pp. 352367, 2015.

[7] Q. J. Xia, M. Rao, Y. Q. Ying, X. M. Shen. “Adaptive fading Kalman filter with an application,” Automatica, vol. 30, pp. 1333-1338, 1994.

[8] C. HAJIYEV, F. CALISKAN. "Sensor and control surface/actuator failure detection and isolation applied to F - 16 flight dynamic,” Aircraft Engineering and Aerospace Technology, vol. 77, pp. 152-160, 2005. 